\title{
Minimum grid digital simulation of chronoamperometry at a disk electrode
}

\author{
Dieter Britz ${ }^{\mathrm{a}, *}$, Ole Østerby ${ }^{\mathrm{b}}$, Jörg Strutwolf ${ }^{\mathrm{c}}$ \\ a Department of Chemistry, Aarhus University, 8000 Aarhus C, Denmark \\ ${ }^{\mathrm{b}}$ Department of Computer Science, Aarhus University, 8200 Aarhus N, Denmark \\ c Institute of Organic Chemistry, University of Tübingen, Auf der Morgenstelle 18, 72076 Tübingen, Germany
}

\section{A R T I C L E I N F O}

\section{Article history:}

Received 5 March 2012

Received in revised form 4 June 2012

Accepted 5 June 2012

Available online 14 June 2012

\section{Keywords:}

Digital simulation

Minimum grid

Disk electrode

Finite differences

\begin{abstract}
A B S T R A C T
We seek to find the best ways to achieve a minimum grid with a given target accuracy in the computed current in the simulation of a diffusion limited chronoamperometric experiment at an ultramicrodisk electrode. We present some results for direct discretisation on the cylindrical geometry in $(R, Z)$ space and in transformed coordinates $(\theta, \Gamma)$, comparing four commonly used transformations. In all cases, the use of multi-point spatial derivative approximations are explored to find an optimum. Orthogonal collocation is studied in the two dimensions, and found less efficient than an evenly divided grid and smaller multi-point approximations. The eigenvalue-eigenvector method is studied and found to be relatively inefficient but was useful in providing some information on error waves seen with three of the four transformations used. These error waves are not due to an error propagation, a fact that became clear from the eigenvalue-eigenvector method, which reproduced the waves.
\end{abstract}

(c) 2012 Elsevier Ltd. All rights reserved.

\section{Introduction}

There is constant interest in the digital simulation of electrochemical processes at ultramicroelectrodes of various geometries. The early paper by Flanagan and Marcoux [1] simulated the disk electrode, using the simple explicit method of Feldberg [2]. At the time, the question was in what way the current-time behaviour deviated from that of a plane electrode (Oldham's "shrouded plane" [3]). This was followed by Kakihana et al. [4] (also using the explicit method) and Heinze [5] (an early use of the alternating direction implicit method ADI [6] see the Glossary for abbreviations), still pursuing the deviation.

It was noticed early that the ultramicrodisk and -band have an edge problem of a current density singularity, both experimentally [7] and theoretically or by simulation [1,3,8-21], and the singularity is implicit in Saito's 1968 work [22], which is the source for the steady state value of the current at a disk (although Saito did not remark on the edge effect). The edge effect led Gavaghan [23] to design a mesh for the simulation of the disk that used very unequal intervals, concentrated not only near the disk surface but also at its edge. This type of mesh has been used by others subsequently for the disk itself [24,25], for a microband $[26,27]$, arrays of disks [28], the scanning electrochemical

\footnotetext{
* Corresponding author. Tel.: +45 87155332; fax: +45 86196199.

E-mail addresses: britz@chem.au.dk (D. Britz), oleby@cs.au.dk (O. Østerby), joerg.strutwolf@uni-tuebingen.de (J. Strutwolf).
}

microscope geometry $[29,30]$, the disk recessed into a conical well [31], the conical electrode protruding from an insulating plane [32], the conical tip electrode [33], the disk flush with a spherical surface [34,35], a finite length cylinder [36] and the three-dimensional case of a finite length band [37]. In some cases, such as the disk, band and the disk in a recess with sloping walls, conformal or quasiconformal maps can be used to advantage, and there have been several of these suggested for the disk [38-41] (most of these can also be applied to the band of infinite length) and the disk in a recess with sloping walls [42]. The above list is a small selection of a large number of such works.

There is a need to examine methods of simulation of chronoamperometry at these electrodes with the aim to minimise computation time, because they all lead to two-dimensional discretised grids. In the following, a number of approaches are examined, focusing on the geometry of the circular disk flush with an insulating plane. The results can then be used for other geometries where conformal (or quasiconformal) maps have been found, such as the band electrode and the recessed disk [42] and, in the case of the direct method, for other two-dimensional systems as well.

The following methods were investigated:

1. Moving $m$-point spatial derivative approximations in $(R, Z)$ coordinates;

2. Moving $m$-point spatial derivative approximations in transformed coordinates;

3. Orthogonal collocation $(\mathrm{OC})$;

4. The eigenvalue-eigenvector method. 
Table 1

Transformations from $(R, Z)$ to $(\theta, \Gamma)$.

\begin{tabular}{|c|c|c|c|}
\hline Transf. & $R=$ & $Z=$ & Reference \\
\hline MF & $\sqrt{\left(1+\Gamma^{2}\right)\left(1-\theta^{2}\right)}$ & $\theta \Gamma$ & Morse and Feshbach [47, p. 1292] \\
\hline MWA & $\cos \theta \cosh \Gamma$ & $\sin \theta \sinh \Gamma$ & Michael, Wightman and Amatore [39] \\
\hline $\mathrm{AF}$ & $\left(1-\theta^{2}\right)^{1 / 2} / \cos \Gamma$ & $\theta \tan \Gamma$ & Amatore and Fosset [38] \\
\hline VB & $\cos \theta \cosh \frac{\Gamma}{1-\Gamma}$ & $\sin \theta \sinh \frac{\Gamma}{1-\Gamma}$ & Verbrugge and Baker [40] \\
\hline OAS & $\sin \theta / \cos \Gamma$ & $\cos \theta \tan \Gamma^{1-1}$ & Oleinick, Amatore and Svir [41] \\
\hline
\end{tabular}

All these were computed using finite differences (see later for details), and the first was also run using the finite element package COMSOL Multiphysics ${ }^{\circledR}$.

The example system chosen here is the potential step to a diffusion limiting potential. This is a severe test of any method, because of the sharp initial transient, which causes rather large errors at short times, even with methods that quickly damp errors such as $\mathrm{BI}$ and BDF, which were used here. The advantage of using this test system is that exact reference values for the disk electrode are known $[43,25]$. Other test systems are possible, such as linear sweep voltammetry (LSV) or cyclic voltammetry (CV). For a given number of steps in potential, LSV or CV will use the same cpu time as the same number of steps in the system used here, as there is a fixed coefficient matrix in both cases. This is also the case if first-order homogeneous chemical reactions are involved, the only difference then being in the coefficients. The transformed grids are designed such that there is close spacing near the electrode, which can accommodate rather small reaction layers if present. Very fast reactions and reactions involving concentration fronts in the bulk away from the electrode require other methods, and are not considered here. For second order homogeneous chemical reactions, the coefficients vary with time and then the coefficient matrix must be computed anew at every new step, greatly increasing the cpu usage. It was considered that the results obtained with the potential step will be transferable to systems requiring repeated computation matrices.

\section{Theory}

The reaction used as example here is the simple potential step with the reaction

$\mathrm{Ox}+\mathrm{e}^{-} \rightarrow \operatorname{Red}$

to a potential where the reaction rate is limited by diffusion.

\subsection{Governing equation in cylindrical coordinates}

The disk geometry is illustrated in Fig. 1. Cylindrical coordinates $(R, Z)$ are used, already normalised by the disk radius $a$. The partial differential equation in this system for the potential step experiment is

$\frac{\partial C}{\partial T}=\frac{\partial^{2} C}{\partial R^{2}}+\frac{1}{R} \frac{\partial C}{\partial R}+\frac{\partial^{2} C}{\partial Z^{2}}$

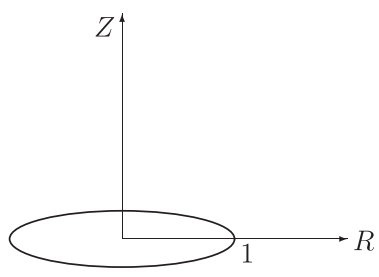

Fig. 1. The disk geometry. in which time $t$ and concentration $c$ are also normalised by

$$
\begin{aligned}
& T=\frac{D t}{a^{2}} \\
& C=\frac{c}{c_{b}},
\end{aligned}
$$

$D$ being the diffusion coefficient and $c_{b}$ the bulk concentration. For a diffusion limited chronoamperometric experiment, the normalised set of boundary conditions is

$T \leq 0$, all $R, Z: \quad C=1$

$T>0, R \leq 1, Z=0: \quad C=0$

$R=0: \quad \frac{\partial C}{\partial R}=0$

$R>1, Z=0: \quad \frac{\partial C}{\partial Z}=0$

$R \rightarrow \infty, Z \rightarrow \infty: \quad C=1$.

Boundary conditions (4c) and (4d) are due to symmetry around these lines, leading to zero flux across these. They can be discretised as such by using one-sided derivative approximations, as is normally done $[38,40,44]$. It has been argued [45, p. 232] and investigated later [46] that these discretisations are not needed, as diffusion can be applied in these places, taking into account the symmetry in the discretisation formulae (see later). This ought to provide better accuracy as more information is made use of. The study [46] concluded that results are identical but in the present study, the zero gradients were nevertheless not made use of directly, for the sake of more accurate computation and to make the eigenvalue-eigenvector method safe to implement.

The above equations and boundary conditions lead to the direct method, that is, discretising the pde (2) directly (and using unequal intervals, see below). However, clearly, this is inefficient compared to using a transformed grid, which will be described. The direct method is included here because for some geometries, no transformations have been found and the direct method is then the only one possible, and should be optimised.

\subsection{Governing equation in transformed coordinates}

Several transformations were compared in this work, as tabulated in Table 1 . In all cases, the new coordinates are $(\theta, \Gamma)$. The transformation in Morse and Feshbach (MF) [47] has been used in only one electrochemical study [48] to our knowledge. It leads to a rather simple and elegant new pde and does concentrate points in the critical area around $(R, Z)=(1,0)$ but unfortunately it was found to perform very poorly compared to the others and will not be mentioned further here.

Four commonly used transformations: those due to Michael, Wightman and Amatore (MWA) [39], Amatore and Fosset (AF) [38], Verbrugge and Baker (VB) [40] and Oleinick, Amatore and Svir(OAS) [41] were compared. VB was found to be the most efficient, closely matched by OAS. 
Table 2

pde coefficients and other quantities for the transformations. For the current integrals $I$, $C_{\Gamma}$ denotes $\partial C /\left.\partial \Gamma\right|_{\Gamma=0}$.

\begin{tabular}{|c|c|c|c|c|}
\hline Quantity & MWA & $\mathrm{AF}$ & VB & OAS \\
\hline$F$ & $\sin ^{2} \theta+\sinh ^{2} \Gamma$ & $\theta^{2}+\tan ^{2} \Gamma$ & $\sin ^{2} \theta+\sinh ^{2}\left(\frac{\Gamma}{1-\Gamma}\right)$ & $\frac{\cos ^{2} \theta+\sin ^{2} \theta \sin ^{2} \Gamma}{\cos ^{2} \Gamma}$ \\
\hline$a_{\theta}$ & 1 & $1-\theta^{2}$ & 1 & 1 \\
\hline$b_{\theta}$ & $-\tan \theta$ & $-2 \theta$ & $-\tan \theta$ & $\cot \theta$ \\
\hline$a_{\Gamma}$ & 1 & $\cos ^{2} \Gamma$ & $(1-\Gamma)^{4}$ & $\cos ^{2} \Gamma$ \\
\hline$b_{\Gamma}$ & $\tanh \Gamma$ & 0 & $(1-\Gamma)^{2} \tanh \left(\frac{\Gamma}{1-\Gamma}\right)-2(1-\Gamma)^{3}$ & 0 \\
\hline$\theta_{\max }$ & $\frac{\pi}{2}$ & 1 & $\frac{\pi}{2}$ & $\frac{\pi}{2}$ \\
\hline$\Gamma_{\max }$ & $\cosh ^{-1}(1+L)$ & $\cos ^{-1}\left(\frac{1}{1+L}\right)$ & $\frac{\cosh ^{-1}(1+L)}{1+\cosh ^{-1}(1+L)}$ & $\cos ^{-1}\left(\frac{1}{1+L}\right)$ \\
\hline$I$ & $\frac{\pi}{2} \int_{0}^{\pi / 2} \cos \theta C_{\Gamma} \mathrm{d} \theta$ & $\frac{\pi}{2} \int_{0}^{1} C_{\Gamma} \mathrm{d} \theta$ & $\frac{\pi}{2} \int_{0}^{\pi / 2} \cos \theta C_{\Gamma} \mathrm{d} \theta$ & $\frac{\pi}{2} \int_{0}^{\pi / 2} \sin \theta C_{\Gamma} \mathrm{d} \theta$ \\
\hline
\end{tabular}

The transformations above (MWA, AF, VB and OAS) lead to a new pde of the general form

$\frac{\partial C}{\partial T}=\frac{1}{F}\left(a_{\theta} \frac{\partial^{2} C}{\partial \theta^{2}}+b_{\theta} \frac{\partial C}{\partial \theta}+a_{\Gamma} \frac{\partial^{2} C}{\partial \Gamma^{2}}+b_{\Gamma} \frac{\partial C}{\partial \Gamma}\right)$

and Table 2 shows the coefficients. Note that there is an error in [45] in the coefficients for OAS. The ranges for $\theta$ can be either $0, \ldots, 1$ or $0, \ldots, \pi / 2$; the choice has of course an impact on the transport pde and coefficient and current expressions; they have been chosen so as to simplify these expressions. The table includes the maximum values of the transformed coordinates $\theta_{\max }$ and $\Gamma_{\max }$ (see below) and the expression for the current $I$, normalised to the steady state current at a disk, as found by Saito [22].

The boundary conditions are then

$$
\begin{aligned}
& T \leq 0, \text { all } \Gamma, \theta: \quad C=1 \\
& T>0, \Gamma=0: \quad C=0 \\
& \Gamma=\Gamma_{\max }: \quad C=1 \\
& \theta=0: \quad \frac{\partial C}{\partial \theta}=0 \\
& \theta=\theta_{\max }: \quad \frac{\partial C}{\partial \theta}=0 .
\end{aligned}
$$

\subsection{Methods}

\subsubsection{Multi-point discretisations}

Multi-point approximations to the spatial derivatives can be applied to the direct grid in $(R, Z)$ or to the transformed coordinates in $(\theta, \Gamma)$. This has been approached previously [44] but not optimised at the time. The method consists of using an $m$-point approximation to the derivatives across the $(N+1)$-point row or column of the grid, where $m \leq N+1$. For each position $i=0, \ldots, N+1$, this leads to $m$ coefficients, so that for the whole row or column, there is an $(N+1) \times m$ table of such coefficients for each coordinate. For example, along a window of points, for a point indexed with $i$, we find $i_{1}$ and $i_{2}$ such that the window $x_{k}, k=i_{1}, \ldots, i_{2}$ is centred on the point at $i$ for odd $m$ and almost so for even $m$. The derivative approximation is then for general variable $u$

$\frac{\partial u_{i}}{\partial x} \approx \sum_{k=i_{1}}^{i_{2}} b_{p} u_{k}$

where $p=k-i_{1}+1$ because the approximation coefficients are indexed from 1 to $m$. Coordinate $x$ is either $\theta$ or $\Gamma$. For points near the edges at $\theta=0$ or $\theta_{\max }$ (indices 0 or $N_{\theta}$ ), there are several strategies, see Section 3.2.3. The aim here is to optimise both $N$ and $m$, with a target error condition in mind.

The coefficients for the approximations can be obtained easily by using the Fornberg algorithm [49] (both a C++ due to L.K. Bieniasz (private communication) and Fortran90 realisation can be obtained from one of us (DB)). This algorithm is more accurate than the matrix calculation previously described [45, p. 44], that is, it yields accurate derivatives up to higher values of $m$; in this work, $m$ going as high as 30 have been used with good accuracy. The Fornberg algorithm yields $m$ coefficients over a stretch of $m$ points, for derivatives of a desired order (or in fact interpolation if wanted); in the present case, first and second spatial derivatives. These are multiplied by $b_{\theta}$ and $a_{\theta}$ for first and second derivatives respectively along $\theta$, and $b_{\Gamma}$ and $a_{\Gamma}$ for first and second derivatives along $\Gamma$, seen in (5).

\subsubsection{Orthogonal collocation}

OC is a special case of multi-point approximations, where $m=N+1$, that is, all points in a row or column are used, and the points are distributed as the roots of certain polynomials, often chosen as Jacobi polynomials [50], that exhibit close spacing at either end of the row. Jacobi polynomial roots require cumbersome computation, and Chebyshev polynomial roots were found to perform equally well. Speiser and Pons applied the method to a two-dimensional simulation [17,18], as did Parikh and Liddel [51-53]. Descriptions of OC are normally somewhat heavy on linear algebra but in fact OC, whether in one or more dimensions, can be simply implemented by the derivative approximation formula applied to a stretch of points at $x_{j}, j=0, \ldots, N$, for the roots of Chebyshev polynomials shifted to the range $[0,1]$

$x_{0}=0$

$x_{j}=\frac{1}{2}\left(1-\cos \phi_{j}\right) \quad\left(j=1, \ldots, N-1, \phi_{j}=\frac{\pi(2 j-1)}{2(N-1)}\right)$

$x_{N}=1$

and scaling to the length desired. The application of the Fornberg algorithm to all $N+1$ points of the row and column (each with its own range) results in two $(N+1) \times(N+1)$ matrices for each of the first and second derivatives. These are however combined in practice, see below.

\subsubsection{Eigenvalue-eigenvector method}

There is a good description of this method in Smith [54], which is the basis of the present implementation. The method was introduced into electrochemical simulations by Friedrichs et al. [55], followed by one other work [56]. It is also described in [45, p. 182]. The method starts with the discretisation only of the space variables in (5), leaving the left-hand side as it is. This generates a system of ordinary differential equations. We anticipate here the computational information to be given later, by noting that the two-dimensional grid of concentration points on $(\theta, \Gamma)$ is unfolded into a one-dimensional vector of unknowns $\mathbf{u}$, using a suitable mapping function. This yields a number $N=\left(N_{\theta}+1\right) \times\left(N_{\Gamma}+1\right)$ of unknowns, unfolded such that the first $N_{\theta}+1$ points are those for the electrode $\left(\Gamma=0, j=0, \ldots, N_{\theta}\right)$ and the last $N_{\theta}+1$ are those for the bulk, $\Gamma=\Gamma_{\max }$, same $j$ range. These are all fixed (at zero and unity, 
respectively) and therefore their entry into the system of equations is simply $\partial u_{k} / \partial T=0, k=1, \ldots, N_{\theta}, N-N_{\theta}+1, \ldots, N$. We then have

$$
\frac{\mathrm{d} \mathbf{u}}{\mathrm{d} T}=\mathbf{A u}
$$

with $\mathbf{A}$ being the matrix of coefficients from the space discretisation. The boundary conditions are included in this in that the fixed values are included as mentioned, and the initial values as given by (6a) are represented as $\mathbf{u}_{0}$. The equations at the edge points, $\theta=0$, $\pi / 2$ are discretised as described below and they too have the form $\partial u / \partial T=f(u)$. This is one reason for not using the boundary conditions (6d) and (6e) directly as this does not include a time derivative (although it can be obtained). Eq. (9) has the formal solution

$\mathbf{u}(T)=\exp (T \mathbf{A}) \mathbf{u}_{0}$.

This requires computing the exponential of a matrix [57]. One way of doing this is to use the eigenvalues and eigenvectors of $\mathbf{A}$ provided that $\mathbf{A}$ has a complete set of eigenvectors. If $\mathbf{X}$ is the matrix of all eigenvectors of $\mathbf{A}$ and $\boldsymbol{\Lambda}$ a diagonal matrix with the corresponding eigenvalues in the diagonal then

$$
\mathbf{A X}=\mathbf{X} \mathbf{\Lambda}
$$

or

$$
\mathbf{A}=\mathbf{X} \mathbf{\Lambda} \mathbf{X}^{-1}
$$

and

$\exp (T \mathbf{A})=\mathbf{X} \exp (T \mathbf{\Lambda}) \mathbf{X}^{-1}$

where $\exp (T \boldsymbol{\Lambda})$ is simply the diagonal matrix containing the exponentials of the eigenvalues multiplied by $T$ (see Smith [54] or [45, p. 182] for more details). The method has the disadvantage that all eigenvectors and -values must be computed, but after this, computation of (10) for a number of wanted $T$ is very fast. It is sometimes not necessary to know concentration profiles at a large number of times, so this method allows computing only at those times that are wanted; whereas with a time-marching simulation method, rather small steps in time must be taken in order to achieve a given accuracy. Here, the accuracy depends only on the grid intervals and the precision to which the eigen quantities can be computed. Smith [54] simplifies the method further, by using only the eigenvalue with the smallest magnitude (they have negative real components), because the others do not contribute as much to the solution. This was not used in the present study because once the initial computations are done, the application of (10) is very fast, so no such savings of computer time are needed.

It is worth noting that this method does not involve propagation, neither of the concentration values, nor errors in these, from one step to the next, as happens in time-marching methods such as BI or BDF. The concentration profile for each desired time is computed directly without stepping in discrete time intervals, so there is no propagation. This point becomes relevant below in the context of error waves in time.

\subsubsection{Note on COMSOL}

Due to the ease of use and its versatility, the commercial finite element software package COMSOL Multiphysics has been frequently employed for simulation in electrochemistry, especially for simulation of diffusional transport and kinetics at micro electrodes. To give an impression how COMSOL Multiphysics, developed for a variety of physics and engineering simulations, including coupled phenomena, compares to self-written code optimised for a specific problem, we implemented the UMDE transient problem in COMSOL Multiphysics using the diffusion application mode.

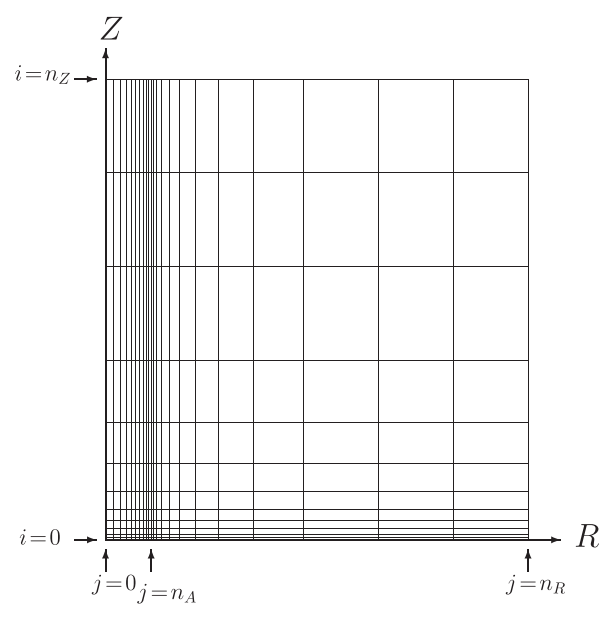

Fig. 2. Coarse example grid from the direct discretisation of (2).

\section{Computational details}

\section{1. $(R, Z)$ space simulations}

Fig. 2 shows the grid used in $(R, Z)$ space. This must have unequal intervals, concentrated both near the electrode in the direction $Z$ and near the disk edge in both directions, as described by Gavaghan [23]. Derivatives are then computed on this uneven grid, but the coefficients for these are, as mentioned above, obtained by using the Fornberg algorithm [49]. This leads to three sets of approximation coefficients, $b_{R}$ and $a_{R}$ for the first and second derivatives, respectively, along $R$, and $a_{Z}$ for the second derivative along $Z$, so that at the grid point indexed with $(i, j)$ we have the semidiscretised form of (2),

$$
\left.\frac{\partial C}{\partial T}\right|_{i, j} \approx \sum_{k=j 1}^{j 2}\left(a_{R}(j, k)+\frac{1}{R_{j}} b_{R}(j, k)\right) C_{i, k}+\sum_{k=i 1}^{i 2} a_{Z}(i, k) C_{k, j}
$$

for all points except the boundary points and the axis. The coefficients for $R$ can be lumped into weighting factors $w_{R}$, leading to

$$
\left.\frac{\partial C}{\partial T}\right|_{i, j} \approx \sum_{k=j 1}^{j 2} w_{R}(j, k) C_{i, k}+\sum_{k=i 1}^{i 2} a_{Z}(i, k) C_{k, j} .
$$

With these coordinates there is the problem of the term in $1 / R$ on the axis, where $R=0$. One could simply use the condition (4c), discretising it as one-sided approximation. It is however better to allow diffusion in this region by using both second spatial derivatives, invoking (4c) only in the sense of symmetry about the axis. The $1 / R$ problem is handled as suggested by Crank and Furzeland [12], using the Maclaurin formula

$\lim _{R \rightarrow 0}\left(\frac{\partial^{2} C}{\partial R^{2}}+\frac{1}{R} \frac{\partial C}{\partial R}\right)=2 \frac{\partial^{2} C}{\partial R^{2}}$

which eliminates the axis problem. The $m$ points then straddle the axis as described above for the left- and right-hand edges of the transformed grids, for example (25).

There is a need to set spatial limits to the grid, making use of the limit quantity $L$. This is normally set to the value $6 \sqrt{T_{\max }}$ since the seminal paper by Feldberg in 1969 [2], $T_{\max }$ being the dimensionless maximum time. For the Cottrell simulation in one dimension, this factor leads to an outer boundary at which during the observation time $T_{\max }$, there are no changes in concentration greater than $10^{-4}$ relative to the bulk concentration. To ensure better accuracy in the present study, $L$ was always set equal to $8 \sqrt{T_{\max }}$. 
For the cylindrical space in $(R, Z)$ maximum values $R_{\max }$ and $Z_{\max }$ are

$R_{\max }=1+L$

$Z_{\max }=L$.

The computational domain for the COMSOL simulations is the same as for the finite difference simulations and given by Eq. (17). Triangular elements are used for creating the mesh.

Fixed spatial grids were used. Adaptive grids are possible and have been used, for example by Harriman et al. [58-63] and Nann and Heinze [64] for two-dimensional systems, but are of advantage only in $(R, Z)$ space. In transformed space, there is very close spacing where it is needed, near the electrode, accommodating almost all applications except sharp concentration changes away from the electrode, which are a special area of study, not dealt with in the present work.

\subsection{Transformed coordinates}

The grids in $(\theta, \Gamma)$ transformed coordinates are divided into respectively $N_{\theta}$ and $N_{\Gamma}$ intervals, evenly except in the case of OC, where the grid points are placed as the roots of Chebyshev polynomials, as given above, in both directions and scaling to the maximum values. In this case, we have both first and second spatial derivatives in both coordinates, leading to lumped coefficients and a semidiscretised form at grid point $(i, j)$

$$
\left.\frac{\partial C}{\partial T}\right|_{i, j} \approx \sum_{k=j 1}^{j 2} w_{\theta}(j, k) C_{i, k}+\sum_{k=i 1}^{i 2} w_{\Gamma}(i, k) C_{k, j},
$$

$w_{\theta}$ and $w_{\Gamma}$ being composites of the coefficients $a_{\theta}, b_{\theta}$ and $a_{\Gamma}, b_{\Gamma}$.

\subsubsection{Limits}

The maximum time to which the simulation is to be driven is $T_{\max }$. Two values of $T_{\max }$ were chosen as examples: 1 and 50 . The former means that a simulation is driven to some observation time, while the latter is typically the number of dimensionless time units for a cyclic voltammetry scan, if the (dimensionless) potential range covers 24 units, forward, and backwards.

For the spatial limits in the case of transformed coordinates, see Table 2 . These are explained in [45, p. 229] but essentially ensure limits equivalent to those described for cylindrical coordinates, in (17).

\subsubsection{Time discretisation}

The pde

$\frac{\partial C}{\partial T}=\nabla^{2} C$

is now discretised in time. The simplest method is BI [65] which gives

$$
\frac{C_{k}^{\prime}-C_{k}}{\delta T}=D^{2} C_{k}^{\prime}
$$

or

$-C_{k}=\delta T\left(D^{2} C_{k}^{\prime}-C_{k}^{\prime}\right)$

where $D^{2}$ denotes the discretisation of the right-hand side of (19) as detailed above in (15) and (18) and $C^{\prime}$ denotes the unknown concentration to be found. This can be expressed as the linear system

$$
\mathbf{A C}^{\prime}=\mathbf{B}
$$

where $\mathbf{A}$ is the matrix of coefficients comprising those in Table 2 for the transformed grids and in this case $\mathbf{B}$ is the vector of known concentrations.
$\mathrm{BI}$ is in itself not very efficient, producing errors first-order in time intervals. This is raised to second order by using extrapolation $[66,67]$, and this was used in the present study.

For three-point BDF [68-70], the discretisation is

$$
\frac{{ }^{\prime} C_{k}-4 C_{k}+3 C_{k}^{\prime}}{2 \delta T}=D^{2} C_{k}^{\prime}
$$

or

$$
{ }^{\prime} C_{k}-4 C_{k}=2 \delta T\left(D^{2} C_{k}^{\prime}-3 C_{k}^{\prime}\right)
$$

in which now ' $C$ are the previous concentrations. This leads again to a linear system as above, with $\mathbf{B}$ now being the composite. These linear systems must be solved at each step forward in time. BDF was started by a single BI step to avoid the startup problem that BDF has [71]. Another approach was to use the Douglas-Rachford method [72] but modified [73], which replaces solving a big linear system by solving two tridiagonal systems at each time step.

BDF is also used by COMSOL Multiphysics for the time discretisation. For the current problem COMSOL Multiphysics automatically chooses first order BDF, or BI, to start the simulation. The upper order of BDF is restricted to five.

\subsubsection{Discretisation stencils}

For points near the edges in the coordinate $\theta$, several strategies can be employed, and results are almost identical for timemarching simulations but not for the eigenvalue-eigenvector method, as will be explained. One strategy, which could be called "totally one-sided" is to use discretisation stencils wholly contained within the grid. For a given $m$, this means the range of the stencil for points near the edge at $\theta=0$ will be $\left(j_{1}, j_{2}\right)=(0, m-1)$ up to an index equal to $(m-1) / 2$ (integer division). For larger indices, both $j_{1}$ and $j_{2}$ will move along the range, centring the stencil on each index. As the stencil approaches the right-hand edge at $\theta=\theta_{\max }$, a few points up to that at $j=N_{\theta}$ will share the same stencil $\left(j_{1}, j_{2}\right)=\left(N_{\theta}-m+1\right.$, $\left.N_{\theta}\right)$. At both $\theta=0$ and $\theta_{\max }$, the first derivative is set equal to zero due to (6d) and (6e), and at all interior points we have approximations for both first and second derivatives. Note that even though the same stencil may be used for different indices near the edges, the coefficients will not be the same, as they depend on the reference index.

Another approach may be called "partly one-sided"; it makes use of one-sided stencils for points near, but not on, the edges as above, but on the edges only indirectly uses (6d) and (6e). Here, the sequence of positions on the grid, $\theta_{0}, \theta_{1}, \ldots, \theta_{N}$ is extended beyond both edges to mirror positions, leading to an augmented sequence $\theta_{-p}, \theta_{-p+1}, \ldots, \theta_{0}, \theta_{1}, \ldots, \theta_{N}, \theta_{N+1}, \ldots, \theta_{N+p}$ where $p=(m-1) / 2$. The positions are mirror images of those inside the grid, so that $\theta_{-1}=-\theta_{1}$, etc. and symmetry dictates that $C_{-\theta}=C_{\theta}$. The stencil for the index 0 and $N_{\theta}$ then straddles that edge symmetrically for odd $m$ and nearly so for even $m$, and the routine of Fornberg computes coefficients for the second derivative (there being no first). Those coefficients that fall outside the grid are then added to their mirror image positions inside the grid, and the approximation finally rests on a smaller number of points. For example, if $m=5$ then the five computed coefficients $b_{-2}, \ldots, b_{2}$ lead to

$$
\begin{aligned}
\frac{\partial^{2} c}{\partial \theta^{2}} & \approx b_{-2} C_{-2}+b_{-1} C_{-1}+b_{0} C_{0}+b_{1} C_{1}+b_{2} C_{2} \\
& =b_{0} C_{0}+\left(b_{-1}+b_{1}\right) C_{1}+\left(b_{-2}+b_{2}\right) C_{2}
\end{aligned}
$$

so that here $j_{1}, j_{2}=0$ and 2 , respectively. This is justified by the fact of symmetry around the edges. For indices close to but not on the edges, one-sided stencils can then be used, the index stretching from 0 to $m$.

The two above approaches have been compared [46] and found to lead to the same results. It might be argued that the partly 
one-sided approach makes use of more information than the totally one-sided, but this does not appear to make a difference.

The third approach, here called "straddling" uses stencils straddling the edges for all indices close to the edges and might be regarded as the most rational. As with the partly one-sided, the sequence of positions along $\theta$ is extended at both ends, and for indices up to $(m-1) / 2$ away from the edges, straddling stencils are used, folding the mirrored coefficients into grid points as above. As an example, again for $m=5$, at index 1 we would have

$$
\begin{aligned}
& \frac{\partial^{2} C}{\partial \theta^{2}} \approx b_{-1} C_{-1}+b_{0} C_{0}+b_{1} C_{1}+b_{2} C_{2}+b_{3} C_{3} \\
& \quad=b_{0} C_{0}+\left(b_{-1}+b_{1}\right) C_{1}+b_{2} C_{2}+b_{3} C_{3}
\end{aligned}
$$

etc. and similarly for the other edge at $\theta_{\max }$. For even $m$, the stencils were chosen such that there was one more point inside the grid than outside it; however, even $m$ were generally avoided.

\subsubsection{Computers and methods}

Independent computations were performed by the three authors, to ensure correctness. They were run (i) under Linux on a Dell PowerEdge R210 with Xenon X3430 processor using FreePascal and MATLAB [74]; (ii) under Windows 7 (64 bits) on a PC equipped with an AMD Athlon ${ }^{\circledR}$ II X4 processor, using the gfortran 95/2003 compiler V.4.6 and (iii) under Linux running on an Amitech Blueline PC and Intel Fortran 90/95 V.11.3. All computations were run using IEEE 754 Standard double precision, giving approximately 16-decimal precision. The time marching simulations were done using, for the time integration, three-point backward differentiation formula (BDF) [68-70] started with one backwards implicit or Laasonen [65] (BI) step; and by BI with extrapolation [66,67,75]; as well as by the Douglas-Rachford method [72,73]. All three methods produced very similar results and those for BI/BDF are those presented in the tables. For the time-marching simulations, the discretisations result in sparse banded systems of equations, for which the sparse solver MA28 [76,77] was initially used (but see later). For the eigenvalue-eigenvector method, the eigenvalues and -vectors were computed directly from the whole matrices using the routine RGG [78], converted to Fortran 90. There are Fortran packages available for sparse matrices, but these have such large overhead in terms of computing time that they do not recommend themselves for the relatively modest-sized matrices encountered with the eigenvalue-eigenvector method as used here.

In all time-marches, a (dimensionless) time interval of 0.01 was set, having been found to be sufficient, in the sense that then, only the grid dimensions determined the simulation accuracy.

Errors in the current are reported as relative to exact known values, which were obtained using the formulae of Mahon and Oldham [43] directly, changing from the short-time formula to the longtime one at $T=1$, and also by making use of interpolation between the tabulated current values from [25] in the region where the Mahon-Oldham formulae cross over, to avoid the (very) small sharp change in the computed error at a crossover point. An error target of $0.1 \%$ was set. This may appear too small, given the instrumental accuracy of current measurement, but it was decided to target an error that was smaller than practical measurement for safety. It was decided that this error must be satisfied over the range $\left[T_{\max } / 10\right.$, $\left.T_{\max }\right]$. This time range is somewhat arbitrary, and a different, for example larger, range might have been chosen. A referee has suggested using exponentially expanding time intervals, in order to achieve the target errors at shorter times. This has been attempted, and it can indeed work; however, this has two problems. When each step is taken with a new time interval, the matrix of coefficients must be recalculated at every step, and LU-decomposed. This greatly increases cpu use. The fixed interval of size 0.01 was found to be small enough so that errors depend mainly on the spatial grid

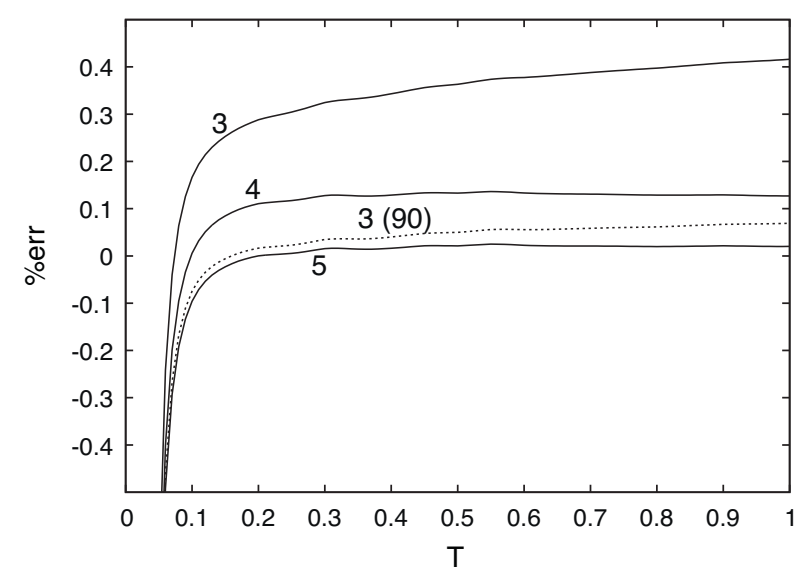

Fig. 3. Relative \% current errors for direct simulations in $(R, Z)$ space, using a grid with $N=60$ over all three regions, and multipoint spatial derivative approximations with marked $m$ values. The dashed line shows errors computed using $N=90$ and $m=3$.

intervals, so smaller time intervals do not help. One must therefore refine the grid. The same benefits of such a finer grid and variable time intervals can be obtained with a finer grid and fixed time intervals, at only slightly increased cpu use. Varying time intervals also render BDF less accurate; the three-point formula used here must be modified. Using BI with extrapolation solves this problem but necessitates extrapolation. Simulations in $(R, Z)$ space were also performed using the finite element program package COMSOL Multiphysics ${ }^{\circledR}$ version 3.5a.

\section{Results and discussion}

Cpu times given in the tables are those for the Amitech computer. They are thus to be taken as relative to each other.

\subsection{Direct simulation in $(R, Z)$ space}

The grids used the same number of exponentially expanding intervals $N$ in $Z$ and $R$ over the disk and in $R$ over the gap between the disk edge and the outer boundary; that is, in practice, $N_{Z}=N_{A}=N_{R}-N_{A}$. This was settled on after some exploratory computations. The notation used here is $N_{Z} \times N_{A}\left(N_{R}\right)$. The simulations yielded some interesting results. Firstly, it was found that there is an optimum minimum interval of $10^{-5}$; if an order of magnitude smaller or larger value is used, accuracy suffers. The problem with a smaller value is that then the expansion factor $\gamma$ [45, p. 105] becomes larger, which degrades accuracy; for larger values, the size of the minimum interval degrades the current approximation. A $\gamma$ value no more than about 1.2 was found best. It was assumed initially that multipoint approximations to spatial derivatives, i.e. $m>3$, would be optimal. Fig. 3 shows plots for a $60 \times 60(120)$ grid, for some marked values of $m$. It appears that from $m=5$, the simulation satisfies the target error expectation, both at the final $T$ but also for the whole range $\left[T_{\max } / 10, T_{\max }\right]$. However, some unexpected features of cpu time needed for the computations emerged, see Table 3. Two things are noteworthy: firstly the very small cpu time for three-point approximations, and secondly the difference in the cpu sequences for even and odd $m$ for the sparse solver, the even $m$ requiring significantly more cpu time than the odd $m$. Both effects are undoubtedly due to the inner workings of the sparse solver MA28. This was confirmed by reprogramming the simulations using a whole-matrix solver, not making use of the sparse nature of the equation system (see the cpu times in the table). These are somewhat irregular but certainly not grouped into even and odd $m$; and the case $m=3$ is not special in this case. 
Table 3

Cpu time for the simulations in $(R, Z)$ (Fig. 3$)$ to $T=1$ on a $60 \times 60(120)$ grid for some values of $m$, solution using both the sparse and the non-sparse solver.

\begin{tabular}{rcc}
\hline$m$ & cpu/s (sparse) & cpu/s (non-sparse) \\
\hline 3 & 0.6 & 24 \\
4 & 16 & 34 \\
5 & 13 & 80 \\
6 & 104 & 103 \\
7 & 41 & 105 \\
8 & 207 & 128 \\
9 & 55 & 129 \\
10 & 434 & \\
\hline
\end{tabular}

The very short cpu time needed for three-point approximations suggests that this may be the optimum, but a larger number of grid intervals is needed for the target accuracy. It was found that a $90 \times 90$ (180) grid, using $m=3$ reached the target, using $1.8 \mathrm{~s}$, still much faster than when using $m=5$ with the coarser grid. The errors are shown as the dashed line in Fig. 3. For larger $T_{\max }=50$, however, a $125 \times 125(250)$ grid was required for $m=3$, using about twice the time needed for $m=5$ and a $60 \times 60$ grid.

As with finite differences, a high number of mesh points close to the electrode edge and surface is desirable. In COMSOL Multiphysics this is achieved by using free mesh parameters instead of the default meshing. These parameters are the maximal sizes of the (triangular) elements along the electrode surface and at the electrode edge as well as expansion factors. There are limits for the values of the maximal elements and the expansion factors. If the maximal element size is too small and/or the expansion factor to high, triangularization of the computational domain is not possible. By trial and error and convergence runs, we found values of $2 \times 10^{-5}$ and $8 \times 10^{-4}$ for the maximal element size at the electrode surface and the edge point and an expansion factor of 1.1 as an optimum. These values are for an $R_{\max } \times Z_{\max }$ domain for $T=1$ (see Eq. (17)). The resulting mesh contains 14215 mesh points and 55015 degrees of freedom.

Performing simulations with a target time of $T=1$ and fixed time steps of 0.01 resulted in an error of $0.52 \%$ at the target time, taking $7 \mathrm{~s}$ of computing time. The computing time is only roughly comparable to the time measured for our own code, since it does not take into account calculation of the current and writing the output to the hard disk, which is done in a post-processing mode. Allowing free time steps controlled by the local error and using default relative and absolute tolerance of 0.01 and 0.001 , respectively, results in a percentage current error of 0.29 at $T_{\max }=1$ using in total 209 time steps. The computing time was $27 \mathrm{~s}$.

\subsection{Comparison of the four transformations}

It is also of interest to compare the different transformation formulae, but this does not appear to have been done previously. Fig. 4 based on runs on a $13 \times 13$ grid with $m=7$ going to $T=1$, provides an indication; AF is clearly the least accurate and VB and OAS perform very similarly. Fig. 5 shows errors over a larger time scale, (but see below for the waves seen there). All methods converge to within the error band of $\pm 0.1 \%$. Thus, all four transformations are useful but $\mathrm{AF}$ and MWA require a finer grid and longer cpu times than the other two.

\subsubsection{Verbrugge-Baker transformation, $V B$}

Using the VB-transformed grid divided into equal intervals, and simulating to $T_{\max }=1$, it was found that a $13 \times 13$ grid could achieve the required accuracy with $m=7$ and 9, as is seen in Fig. 6. Cpu times for the $m$ values $3,4,5,7,9$ were, respectively 4, 8, 8, 20 and $36 \mathrm{~ms}$. The optimum $m$ is 7; smaller and larger values lead to larger errors

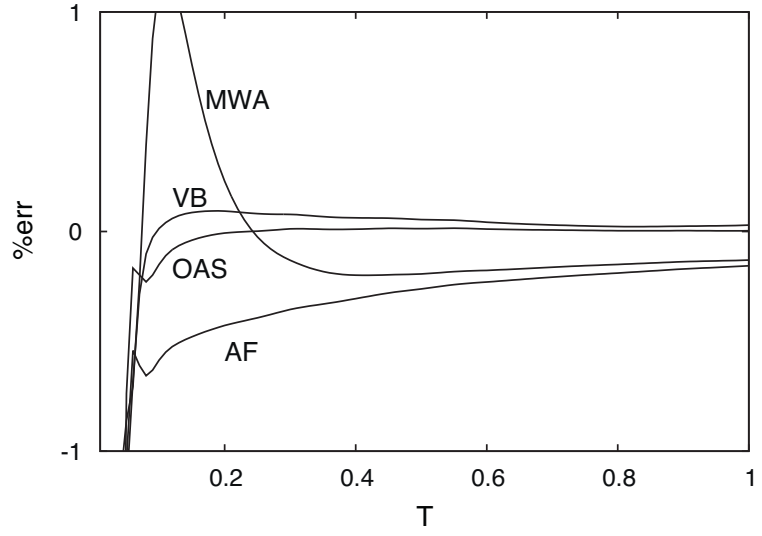

Fig. 4. Comparison of four transformations as marked using, for all, an evenly divided $13 \times 13$ grid and $m=7$.

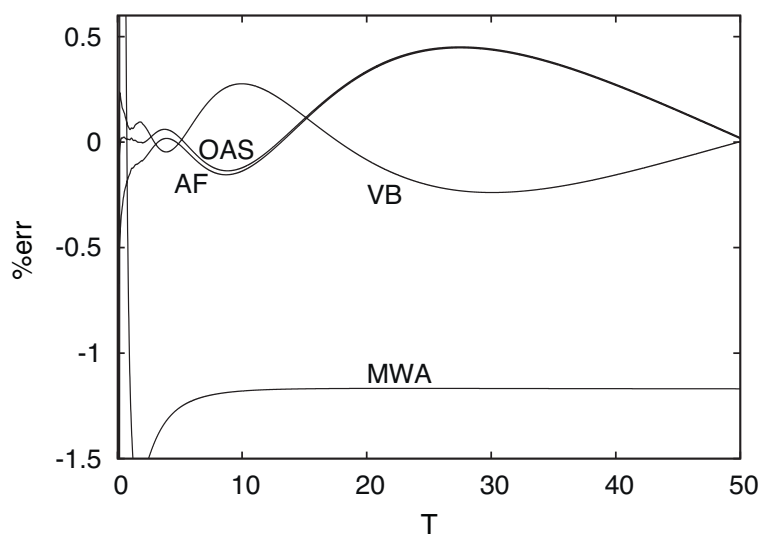

Fig. 5. \% errors vs. $T$ for a $13 \times 13$ grid with $m=7$ for the four different transformations as marked, AF, MWA, OAS and VB (see text).

or errors that do not satisfy the demand that it holds in the range $0.1 \leq T \leq 1$. Larger $m$ than the optimal result in both larger errors and longer cpu times. For this system there is no special fast execution with three-point approximations.

One might, looking at Fig. 6, assume that for all $m>3$, there has been convergence. However, when the time scale is extended to 50 , we note error waves, see Fig. 7. In that figure, $m=7$ has been used, and various grid dimensions, as marked. Firstly, the waves appear to diverge, which might indicate a slight instability. They do become

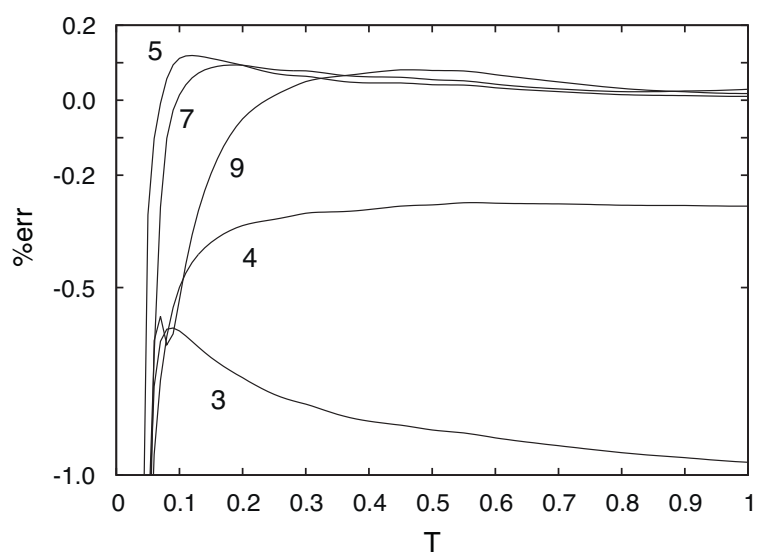

Fig. 6. Relative \% current errors for a grid in $\theta, \Gamma$ (Verbrugge-Baker transformation) using an evenly divided $13 \times 13$ grid, with marked values of $m$. 


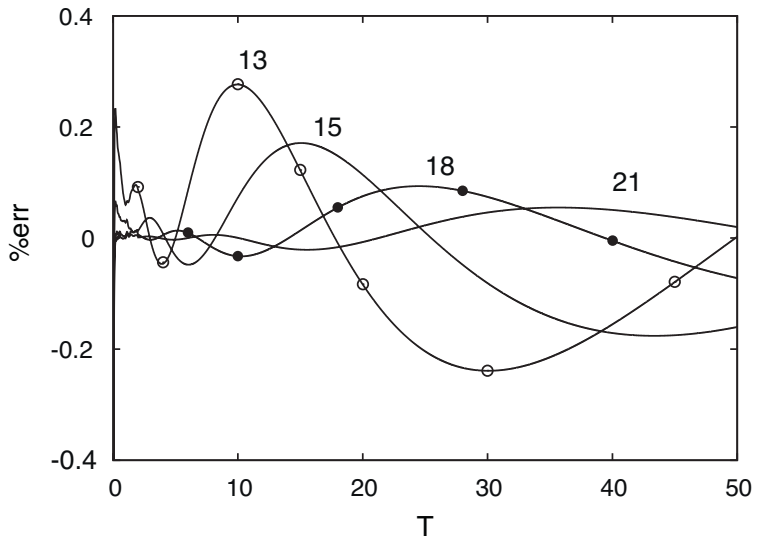

Fig. 7. As Fig. 6 over an extended range of $T$ and with varying grid dimensions as marked (same number of intervals in both directions) and $m=7$. The points on the two curves were computed using the eigenvalue-eigenvector method for the same grid dimensions.

smaller, though still slowly diverging, as the grid is refined. This is not an instability due to an error propagation as one might get using an unstable simulation method; this is shown by the points on two of the curves, obtained by the eigenvalue-eigenvector method, which computes a current directly for each $T$ value, without stepping with time intervals. This is the reason that the word "waves" is used here, rather than "oscillation", such as one obtains with the Crank-Nicolson method [79] applied to problems with an initial transient $[80,81]$, which are indeed oscillatory error propagation effects. We have no explanation for this puzzling effect, which is clearly a property of the transformed grid. The three transformations AF, OAS and VB all produce waves, but MWA does not, converging to a constant error at large $T$ (see Fig. 5), confirming that the errors are a function of the grid. Fig. 7 also shows that in order to get the target error band, an $18 \times 18$ grid is needed. For larger $T_{\max }$ still, more grid points would be needed.

\subsection{Using whole-matrix solutions}

The sparse solver package MA2 8 has quite some overhead and for smallish matrices, it it often better to ignore the sparsity of the linear system and use a non-sparse solver. This was investigated for the VB grid for some grid dimensions and the results are shown in Table 4. Even for grids as large as $30 \times 30$, which are larger than needed, the non-sparse solver is faster than the sparse solver. This is convenient for the programmer. In the case of $(R, Z)$ coordinates, the situation is not as clear. Table 3 shows that for the $60 \times 60$ grid, the non-sparse solver is faster for $m=6$ and 8 , where the sparse solver was seen to be slow. In that case, it was found (above) that $m=3$ is particularly fast but requires a finer grid for the target accuracy, that is, a $90 \times 90(180)$ grid. The system to be solved has 16471 unknowns, which requires much memory of the computer. It was possible to do this with the non-sparse solver directly in $(R, Z)$ but it required $945 \mathrm{~s}$ cpu time, and a day or so of real time, as there

Table 4

Cpu times using the sparse and the non-sparse solver for a VB grid of stated dimensions, simulating with steps 0.01 to $T=1$. For all, $m=7$.

\begin{tabular}{lll}
\hline Grid size & Cpu/s sparse & Cpu/s non-sparse \\
\hline $13 \times 13$ & 0.024 & 0.013 \\
$15 \times 15$ & 0.040 & 0.021 \\
$18 \times 18$ & 0.076 & 0.041 \\
$21 \times 21$ & 0.132 & 0.082 \\
$25 \times 25$ & 0.280 & 0.188 \\
$30 \times 30$ & 0.572 & 0.400 \\
\hline
\end{tabular}

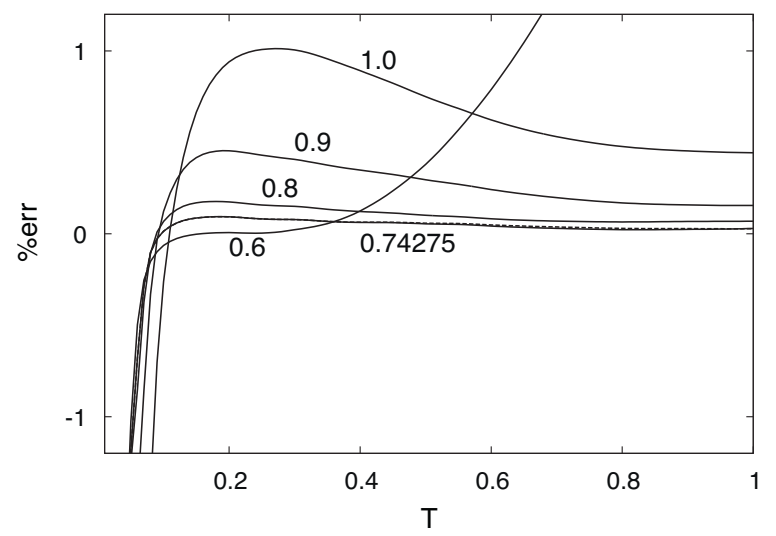

Fig. 8. Effect of varying $\Gamma_{\max }$ for the VB transformed $13 \times 13$ grid, $m=7$. $\Gamma_{\max }=0.74275$ is the computed $\Gamma_{\max }$ value, and the dashed line is for the same interval $(0.74275 / 13)$ but with 17 intervals, making $\Gamma_{\max }=0.971$. This line is barely distinguishable from that for 13 intervals and the computed $\Gamma_{\max }$.

was a great deal of disk swapping, understandably. Against this, the sparse solver needed $1.7 \mathrm{~s}$ for the same calculation. So, generally, for the direct simulation in $(R, Z)$, a sparse solver such as MA28 is advisable.

\subsubsection{Spatial limits}

When working on the direct grid in $(R, Z)$, the spatial limits are easy to define, as given by (17). When using MWA or AF, it is necessary to transform these limits to appropriate transformed coordinates. In all cases including VB, the variable $\theta$ has set limits, either $[0,1]$ or more conveniently $[0, \pi / 2]$ (which simplifies the governing equations), but a maximum $\Gamma_{\max }$ must be computed as given in Table 2 . It is a function of the chosen $T_{\max }$. VB spans the whole semiinfinite diffusion space for $0 \leq \Gamma \leq 1$, that is, $\Gamma_{\max }=1$. It was however found that using a $\Gamma_{\max }$ value as computed from the formula in the table results in significantly smaller errors, even though $\Gamma_{\max }$ is not much less then unity. The same result could be achieved by increasing the number of intervals in $\Gamma$ while fixing their lengths, up to close to unity, showing that the interval size, and a sufficient number of them, is crucial. For efficiency, the computed value of $\Gamma_{\max }$ is best, as it requires the smallest $N_{\Gamma}$. Fig. 8 shows this effect. Note the dramatic effect of a $\Gamma_{\max }$ value that is too small. In that case, the diffusional space is too small and the constant boundary condition (6c) is not appropriate and leads to larger errors.

\subsection{Orthogonal collocation}

Here, the whole width and height of the grid is used for the polynomial, that is, $m_{\theta}=N_{\theta}+1$ and $m_{\Gamma}=N_{\Gamma}+1$, the two not necessarily being the same (although in practice they were chosen equal). The nodes were chosen as the roots of Chebyshev polynomials as explained above, scaling to the ranges $\Gamma_{\max }$ and $\theta_{\max }$. Fig. 9 shows the result for $T_{\max }=1$ and it is seen that we need a grid of at least $14 \times 14$ for our target accuracy in the specified range, requiring $0.044 \mathrm{~s}$ of cpu time. It was found that in general, this method required more cpu time than using evenly spaced points and a smaller $m$, although for larger $T_{\max }$, the difference is not very great.

\subsection{Eigenvalue-eigenvector method}

The eigenvalue-eigenvector method was not used in the case of the direct system in $(R, Z)$, there being too many nodes.

A curious phenomenon was found here. Initially, the discretisations were applied in the manner called "partly one-sided" in 
Table 5

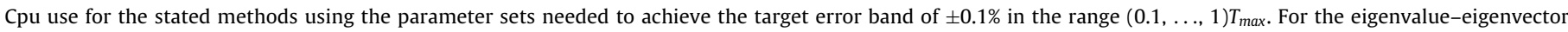

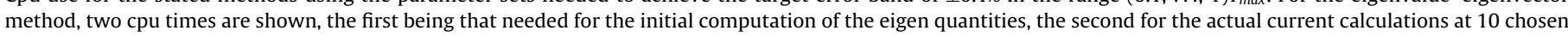
$T$ values.

\begin{tabular}{|c|c|c|c|c|c|}
\hline Method (coordinate system) & $T_{\max }$ & Grid dimensions & $m$ & $\mathrm{cpu} / \mathrm{s}$ & Figure \\
\hline$(R, Z)$ & 1 & $60 \times 60(120)$ & 5 & 13 & \\
\hline$(R, Z)$ & 50 & $60 \times 60(120)$ & 5 & 36 & \\
\hline$(R, Z)$ & 1 & $90 \times 90(180)$ & 3 & 1.8 & Fig. 3 \\
\hline$(R, Z)$ & 50 & $125 \times 125(250)$ & 3 & 63 & \\
\hline $\mathrm{VB}(\theta, \Gamma)$ sparse & 1 & $13 \times 13$ & 7 & 0.024 & Fig. 6 \\
\hline $\mathrm{VB}(\theta, \Gamma)$ sparse & 50 & $18 \times 18$ & 7 & 0.53 & Fig. 7 \\
\hline VB as above, whole-matrix & 1 & $13 \times 13$ & 7 & 0.013 & \\
\hline VB as above, whole-matrix & 50 & $18 \times 18$ & 7 & 1.4 & \\
\hline VB with orthogonal collocation & 1 & $14 \times 14$ & $(15)$ & 0.044 & Fig. 9 \\
\hline VB with orthogonal collocation & 50 & $16 \times 16$ & $(17)$ & 0.62 & Fig. 10 \\
\hline $\mathrm{VB}$, eigenvalue-eigenvector method & 1 & $14 \times 14$ & 7 & $0.5,0.004$ & \\
\hline $\mathrm{VB}$, eigenvalue-eigenvector method & 50 & $18 \times 18$ & 7 & $2.2,0.020$ & Fig. 7 \\
\hline
\end{tabular}

Section 3.2.3, using only a straddling stencil on the edges of $\theta$. It was found that for rather large $N$ (in fact, larger than needed for accurate results), eigenvalues with positive real components appeared, rendering the method impossible, note Eqs. (10) and (13). It was decided then to use the "straddling" stencil discretisation, and this removed the problem; all eigenvalues now had negative or zero real components. This may be seen as somewhat academic, because the problem only arose for $N$ that were larger than needed, but it was considered that it is better to be on the safe side.

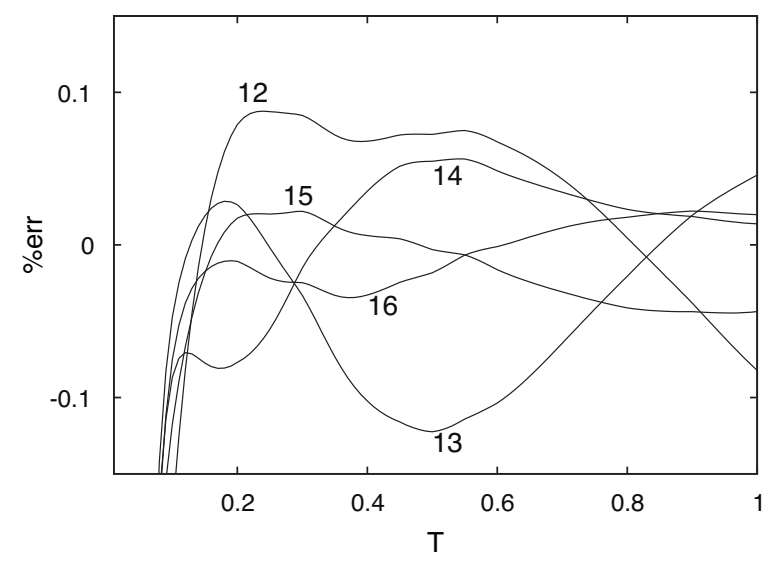

Fig. 9. Orthogonal collocation: Relative \% current errors for a grid in $\theta, \Gamma$ (Verbrugge-Baker transformation) using a grid with point distribution according to the roots of Chebyshev polynomials with marked values of $N$ (equal in both coordinates), for $T_{\max }=1$. Of necessity, $m=N+1$.

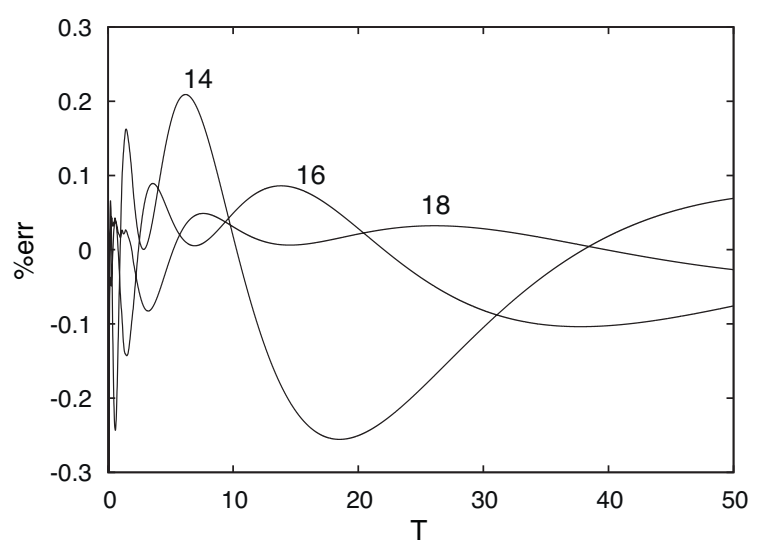

Fig. 10. As Fig. 9 over an extended range of $T$ and with varying grid dimensions as marked.
The method produces almost the same current values as the time-march (there is no contribution from the time intervals here). It requires significantly more cpu time during the computation of the eigenvalues and -vectors than the time-march. After this phase, however, computing currents at a number of $T$ values is very fast. For small times, the method produced slightly larger errors than a corresponding time march, and for $T_{\max }=1$, where a time march required a $13 \times 13$ grid, the eigen-method required one of size $14 \times 14$ to achieve the error target. At larger $T_{\max }$, no differences were found between the methods.

All in all, the eigenvalue-eigenvector method appears to be inefficient. In the context of the present paper it was useful in the sense that it produced the points indicated in Fig. 7, confirming that the error waves seen in the time march do not stem from the time march itself as an error propagation effect but must be a property of the grid when the transformations AF, VB and OAS are used.

\subsection{Cpu times and efficiency}

Table 5 shows a summary of the cpu times required for the various methods with the parameters required for our target error band. These are relative to each other, as a more modern computer would no doubt be faster, but do provide a comparison between the methods. When $T_{\max }=1 \mathrm{VB}, m=7$, non-sparse is fastest, followed by sparse and OC. When $T_{\max }=50 \mathrm{VB}, m=7$, sparse is fastest, followed closely by OC. OC does not however have any advantage over an equally divided grid and a smaller $m$. Working in $(R, Z)$ space directly if that is the choice, it is possible to get down to cpu times of a few seconds or tens of seconds. The choice may be mandated, if the geometry is one for which no transformation has been found.

\section{Conclusions}

A general purpose simulation package as COMSOL Multiphysics might not be as efficient as specifically written code for the relatively simple problem treated here. However, in addition to the computing time alone, one might also consider the amount of time which goes into writing and debugging program code. Using the graphical user interface of COMSOL Multiphysics, the diffusion problem is specified within a couple of minutes. However, the real strength of COMSOL Multiphysics comes into play when solving coupled phenomena, for example diffusion and momentum transport, which was not the subject here.

Considering Table 5, clearly the most efficient method is a timemarching method such as BI or BDF on a grid transformed with the Verbrugge-Baker conformal map [40], taking the least cpu time for a grid that is sufficiently fine so as to satisfy our target error 
criteria. This was achieved using a grid in $(\theta, \Gamma)$ only $13 \times 13$ with $m=7$. Larger values of $m$ did not improve the results, rather the reverse, as oscillations set in for these. Direct solution of the linear system, not taking into account the sparse nature of the linear system, is most efficient here, due to the computational overhead of the sparse solver used. The three methods, BDF, BI and Douglas-Rachford performed very similarly, producing almost identical errors.

If one wants to discretise directly on the grid in $(R, Z)$ space, then reasonably short cpu times can be achieved using grid point distributions with exponentially expanding intervals in the $Z$ direction from the electrode and in both directions away from the vertical line $R=1$. There is an optimum smallest base interval, below and above which the computation of the total current becomes less accurate; generally, an expansion factor no greater than about 1.2 is best. For this system, a sparse solver such as MA2 8 should be used to solve the linear system at each iteration in time. In this study, target errors were achieved either using 60 intervals in all three regions in this space (the $Z$ range, the $R$ range above the electrode and that above the insulating area beyond the electrode), with $m=5$ (13 s), but a finer grid with $N=90$ and $m=3$ was even faster $(1.8 \mathrm{~s})$, due to the fact that the sparse solver becomes very fast for that $m$. Going to $T_{\max }=50$, we have a choice of a $60 \times 60(120)$ grid with $m=5(36 \mathrm{~s})$ or a $125 \times 125(250)$ grid and $m=3(63 \mathrm{~s})$.

Even when using VB, it is beneficial to set the value of $\Gamma_{\max }$ to that calculated by the formula in Table 2 , which provides significantly smaller current errors than setting $\Gamma_{\max }=1$.

Orthogonal collocation was found not to be as efficient as an even distribution of the grid points and an $m$ smaller than the grid dimensions in both directions, so this method does not have an advantage over straight VB.

Using the transformations $\mathrm{AF}, \mathrm{VB}$ and $\mathrm{OAS}$, waves are observed in the current errors. Computations with the eigenvalue-eigenvector method, which does not involve discretisation in time and therefore does not involve error propagation (in time), showed that these waves are not due to instability but must be a result of some grid property, that is the discretisation using these transformations. In all these cases, the waves elicit what appears as a slight divergence in time, which can be made smaller by refining the grid. With MWA, there are no such waves, the current error converging, with time, on a constant value, which also can be made smaller by grid refinement. To our knowledge, these waves have not been remarked on previously and it is not clear what causes them. They do not appear when discretising directly in $(R, Z)$.

Finally, the eigenvalue-eigenvector method is relatively inefficient. The method does have the perhaps desirable feature that only those current values at a chosen set of times need to be computed, rather than (as here) in steps of $\delta T=0.01$. For coarse grids, the target errors are not attained and upon refinement of the grid to parameters known to be sufficient from a time-march with VB, the method requires rather large cpu times. The discretisation method, that is, the placement of discretisation stencils, is critical with this method and straddling stencils should be used for points near the edges along $\theta$ to avoid problems of eigenvalues with positive real components.

In the present context, the method was useful in confirming the error wave phenomenon and the fact that it is not due to an error propagation. Also, it is applicable only in those rare cases where the linear system has constant coefficients for all times, because the computation of the eigenvalues and eigenvectors require the most cpu time and if they had to be computed separately for each chosen time, the method would become even more inefficient. This would be the case, for example, for a simulation where the boundary conditions change with time, such as variable time steps or if the system involves second order homogenous chemical reactions.

\section{Appendix A. Nomenclature}

a disk radius

c concentration

$c_{b} \quad$ bulk concentration

$c$ dimensionless concentration $c / c_{b}$

$C_{i, j} \quad$ dimensionless concentration at indices $i$ and $j$

I dimensionless flux, see Table 2

$R \quad$ dimensionless radial coordinate

$T$ dimensionless time, see (3)

$\delta T \quad$ dimensionless time step length

Z dimensionless axial coordinate

A coefficient matrix

B RHS vector as in (22)

$\mathbf{X}$ matrix of eigenvectors

$\boldsymbol{\Lambda} \quad$ matrix of eigenvalues (on the diagonal)

$\theta \quad$ transformed coordinate, see Table 1

$\theta_{\max } \quad \operatorname{maximum} \theta$, see Table 2

$\Gamma \quad$ transformed coordinate, see Table 1

$\Gamma_{\max } \quad \operatorname{maximum} \Gamma$, see Table 2

\section{References}

[1] J.B. Flanagan, L. Marcoux, Digital simulation of edge effects at planar disc electrodes, Journal of Physical Chemistry 77 (1973) 1051.

[2] S.W. Feldberg, Digital simulation: a general method for solving electrochemical diffusion-kinetic problems, in: A.J. Bard (Ed.), in: Electroanal. Chem., vol. 3, Marcel Dekker, New York, 1969, p. 199.

[3] K.B. Oldham, Edge effects in semiinfinite diffusion, Journal of Electroanalytical Chemistry 122 (1981) 1.

[4] M. Kakihana, H. Ikeuchi, G.P. Satô, K. Tokuda, Diffusion current at microdisk electrodes - application to accurate measurement of diffusion coefficients, Journal of Electroanalytical Chemistry 117 (1981) 201.

[5] J. Heinze, Diffusion processes at finite (micro) disk electrodes solved by digital simulation, Journal of Electroanalytical Chemistry 124 (1981) 73.

[6] D.W. Peaceman, H.H. Rachford, The numerical solution of parabolic and elliptic differential equations, Journal of the Society for Industrial and Applied Mathematics 3 (1955) 28.

[7] R.C. Engstrom, C.M. Pharr, M.D. Koppang, Visualization of the edge effect with electrogenerated chemiluminescence, Journal of Electroanalytical Chemistry 221 (1987) 251

[8] A.J. Bard, J.A. Crayston, G.P. Kittlesen, T.V. Shea, M.S. Wrighton, Digital simulation of the measured electrochemical response of reversible redox couples at microelectrode arrays: consequences arising from closely spaced ultramicroelectrodes, Analytical Chemistry 58 (1986) 2321.

[9] J.F. Cassidy, S. Pons, A.S. Hinman, B. Speiser, Simulation of edge effects in electroanalytical experiments by orthogonal collocation. Part 4: Application to voltammetric experiments, Canadian Journal of Chemistry 62 (1984) 716.

[10] J. Cassidy, J. Ghoroghchian, F. Sarfarazi, S. Pons, Simulation of edge effects in electroanalytical experiments by orthogonal collocation. Part 5: Chronoamperometry at ultramicroelectrode ensembles, Canadian Journal of Chemistry 63 (1985) 3577.

[11] D.K. Cope, The edge effect for planar electrodes, Journal of Electroanalytical Chemistry 439 (1997) 7.

[12] J.Crank, R.M. Furzeland, The treatment of boundary singularities in axially symmetric problems containing discs, Journal of the Institute of Mathematics and its Applications 20 (1977) 355.

[13] D.J. Gavaghan, J.S. Rollett, Correction of boundary singularities in numerical simulation of time-dependent diffusion processes at unshielded disc electrodes, Journal of Electroanalytical Chemistry 295 (1990) 1.

[14] D.J. Gavaghan, How accurate is your two-dimensional numerical simulation? Part 1. An introduction, Journal of Electroanalytical Chemistry 420 (1997) 147.

[15] J. Heinze, M. Störzbach, Electrochemistry at ultramicroelectrodes - simulation of heterogeneous and homogeneous kinetics by an improved ADI-technique, Berichte der Bunsen-Gesellschaft Physical Chemistry Chemical Physics 90 (1986) 1043.

[16] J.C. Myland, K.B. Oldham, The electrochemistry of electrode edges and its relevance to partially blocked voltammetric electrodes, Solid State Electrochemistry 13 (2009) 521

[17] B. Speiser, S. Pons, Simulation of edge-effects in electroanalytical experiments by orthogonal collocation. Part 1: Two-dimensional collocation and theory for chronoamperometry, Canadian Journal of Chemistry 60 (1982) 1352.

[18] B. Speiser, S. Pons, Simulation of edge effects in electroanalytical experiments by orthogonal collocation. Part 2: Theory for cyclic voltammetry, Canadian Journal of Chemistry 60 (1982) 2463.

[19] G. Taylor, H.H. Girault, J. McAleer, Digital simulation of charge transfer to an ultramicrodisk interface, Journal of Electroanalytical Chemistry 293 (1990) 19. 
[20] K.B. Oldham, The rc time "constant" at a disk electrode, Electrochemistry Communications 6 (2004) 210.

[21] O.R. Tutty, The current near the edge of a microelectrode for steady state ec' reactions, Journal of Electroanalytical Chemistry 379 (1994) 519.

[22] Y. Saito, A theoretical study on the diffusion current at the stationary electrodes of circular and narrow band types, Review of Polarography (Japan) 15 (1968) 177.

[23] D.J. Gavaghan, An exponentially expanding mesh ideally suited to the fast and efficient simulation of diffusion processes at microdisc electrodes: 1. Derivation of the mesh., Journal of Electroanalytical Chemistry 456 (1998) 1.

[24] A.S. Barnes, I. Streeter, R.G. Compton, On the use of digital staircase ramps for linear sweep voltammetry at microdisc electrodes: large step potentials significantly broaden and shift voltammetric peaks, Journal of Electroanalytical Chemistry 623 (2008) 129.

[25] D. Britz, K. Poulsen, J. Strutwolf, Reference values of the diffusion-limited current at a disk electrode, Electrochimica Acta 50 (2004) 107, see Erratum, ibid 53 (2008) 8101.

[26] B. Fosset, C.A. Amatore, J.E. Bartelt, A.C. Michael, R.M. Wightman, Use of conformal maps to model the voltammetric response of collector-generator double-band electrodes, Analytical Chemistry 63 (1991) 306.

[27] D. Britz, K. Poulsen, J. Strutwolf, Reference values of the diffusion-limited chronoamperometric current at a microband electrode, Electrochimica Acta 51 (2005) 333, see Erratum, ibid 53 (2008) 7805.

[28] O. Ordeig, C.E. Banks, T.J. Davies, J. del Campo, R. Mas, F.X. Mu noz, R.G. Compton, Regular arrays of microdisk electrodes: simulation quantifies the fraction of 'dead' electrodes, Analyst 131 (2006) 440.

[29] J.L. Amphlett, G. Denuault, Scanning electrochemical microscopy (secm): an investigation of the effects of tip geometry on amperometric tip response, Journal of Physical Chemistry B 102 (1998) 9946

[30] D.G. Georganopoulou, D.J. Caruana, J. Strutwolf, D.E. Williams, Electron transfer mediated by glucose oxidase at the liquid/liquid interface, Faraday Discussions $116(2000) 109$.

[31] D. Britz, J. Strutwolf, Electroanalytical response of an ultramicroelectrode at the bottom of an insulating conical well: digital simulation, Electrochimica Acta 52 (2006) 33.

[32] E.J.F. Dickinson, I. Streeter, R.G. Compton, Chronoamperometry and cyclic voltammetry at conical electrodes, microelectrodes, and electrode arrays: theory, Journal of Physical Chemistry B 112 (2008) 4059.

[33] D. Britz, S. Chandra, J. Strutwolf, D.K.Y. Wong, Diffusion-limited chronoamperometry at conical-tip microelectrodes, Electrochimica Acta 55 (2010) 1272.

[34] I.B.Svir, Simulation of the microdisc problem in spherical co-ordinates. Application to electrochemiluminescence homogeneous analysis, Analyst 126 (2001) 1888.

[35] I.B. Svir, V.M. Golovenko, Simulation of the microdisc problem in spherical coordinates. Application to electrogenerated chemiluminescence, Electrochemistry Communications 3 (2001) 11.

[36] D. Britz, O. Østerby, J. Strutwolf, Reference values of the chronoamperometric response at cylindrical and capped cylindrical electrodes, Electrochimica Acta 55 (20) (2010) 5629.

[37] J. Strutwolf, Computational study of chronoamperometry at rectangular microelectrodes, Electroanalysis 17 (2005) 1547

[38] C.A. Amatore, B. Fosset, Space variables well fitted for the study of steady state and near-steady-state diffusion at a microdisk, Journal of Electroanalytical Chemistry 328 (1992) 21.

[39] A.C. Michael, R.M. Wightman, C.A. Amatore, Microdisk electrodes. Part 1: Digital simulation with a conformal map, Journal of Electroanalytical Chemistry 267 (1989) 33.

[40] M.W. Verbrugge, D.R. Baker, Transient diffusion and migration to a disk electrode, Journal of Physical Chemistry 96 (1992) 4572.

[41] A. Oleinick, C. Amatore, I. Svir, Efficient quasi-conformal map for simulation of diffusion at disk microelectrodes, Electrochemistry Communications 6 (2004) 588.

[42] C. Amatore, A.I. Oleinick, I. Svir, Construction of optimal quasi-conformal mappings for the $2 \mathrm{~d}$ numerical simulation of diffusion at microelectrodes. Part 2: Application to recessed or protruding electrodes and their arrays, Journal of Electroanalytical Chemistry 597 (2006) 77

[43] P.J. Mahon, K.B. Oldham, The transient current at the disk electrode under diffusion control: a new determination by the Cope-Tallman method, Electrochimica Acta 49 (2004) 5041.

[44] J. Strutwolf, D. Britz, Higher-order discretisations in electrochemical digital simulation. Part 5: Application to stationary ultramicrodisk electrode simulation, Journal of Electroanalytical Chemistry 566 (2004) 15.

[45] D. Britz, Digital Simulation in Electrochemistry, 3rd ed., Springer, Berlin, 2005.

[46] D. Britz, Simulation of diffusion limited chronoamperometry at disk electrodes without Neumann boundary conditions on the axis or at the insulating plane, International Journal of Electrochemical Science 3 (2008) 1108.

[47] P.M. Morse, H. Feshbach, Methods in Theoretical Physics, McGraw-Hill, New York, 1953, in 2 volumes.

[48] P.A. Kottke, C. Kranz, Y.K. Kwon, J.-F. Masson, B. Mizaikoff, A.G. Fedorov, Theory of polymer entrapped enzyme ultramicroelectrodes: fundamentals, Journal of Electroanalytical Chemistry 612 (2008) 208.

[49] B. Fornberg, Generation of finite difference formulas on arbitrarily spaced grids, Mathematics of Computation 51 (1988) 699.

[50] L.F. Whiting, P.W. Carr, A simple, fast numerical method for the solution of a wide variety of electrochemical diffusion problems, Journal of Electroanalytical Chemistry 81 (1977) 1.
[51] R.S. Parikh, Orthogonal collocation simulation of the rotating ring disk electrode and its application in the anodic dissolution of chalcocite, Ph.D. Thesis, Washington State University, Pullman, Washing, 1988.

[52] R.S. Parikh, K.C. Liddell, Orthogonal collocation simulation of the rotating ring disk electrode., Journal of the Electrochemical Society 135 (1988) 1703.

[53] R.S. Parikh, K.C. Liddell, Orthogonal collocation simulation of the rotating ring disk electrode. Mass transfer with homogeneous reaction, Journal of the Electrochemical Society 136 (1989) 679.

[54] G.D. Smith, Numerical Solution of Partial Differential Equations, 3rd ed., Oxford University Press, Oxford, 1985

[55] M.S. Friedrichs, R.A. Friesner, A.J. Bard, A new approach to electrochemical simulations based on eigenvector-eigenvalue solutions of the diffusion equations. Part I: Potentiostatic boundary conditions, Journal of Electroanalytical Chemistry 258 (1989) 243.

[56] T.C. Kavanaugh, M.S. Friedrichs, R.A. Friesner, A.J. Bard, A new approach to electrochemical simulations based on eigenvalue-eigenvector solutions of the diffusion equation. Part II: Cyclic voltammetry and heterogeneous kinetics., Journal of Electroanalytical Chemistry 283 (1990) 1.

[57] C. Moler, C. Van Loan, Nineteen dubious ways to compute the exponential of a matrix, twenty-five years later, SIAM Review 45 (2003) 1.

[58] K. Harriman, D.J. Gavaghan, P. Houston, E. Süli, Adaptive finite element simulation of currents at microelectrodes to a guaranteed accuracy. Application to a simple model problem, Electrochemistry Communications 2 (2000) 150.

[59] K. Harriman, D.J. Gavaghan, P. Houston, E. Süli, Adaptive finite element simulation of currents at microelectrodes to a guaranteed accuracy: theory, Electrochemistry Communications 2 (2000) 157.

[60] K. Harriman, D.J. Gavaghan, P. Houston, E. Süli, Adaptive finite element simulation of currents at microelectrodes to a guaranteed accuracy. First-order EC' mechanism at inlaid and recessed discs, Electrochemistry Communications 2 (2000) 163.

[61] K. Harriman, D.J. Gavaghan, P. Houston, E. Süli, Adaptive finite element simulation of currents at microelectrodes to a guaranteed accuracy. An E reaction at a channel microband electrode, Electrochemistry Communications 2 (2000) 567.

[62] K. Harriman, D.J. Gavaghan, P. Houston, D. Kay, E. Süli, Adaptive finite element simulation of currents at microelectrodes to a guaranteed accuracy. ece and $\mathrm{ec}_{2}$ e mechanisms at channel microband electrodes, Electrochemistry Communications 2 (2000) 576

[63] K. Harriman, D.J. Gavaghan, E. Süli, Adaptive finite element simulation of chronoamperometry at microdisc electrodes, Electrochemistry Communications 5 (2003) 519.

[64] T. Nann, J. Heinze, Simulation in electrochemistry using the finite element part 2: scanning electrochemical microscopy, Electrochimica Acta 48 (2003) 3880.

[65] P. Laasonen, Über eine Methode zur Lösung der Wärmeleitungsgleichung, Acta Mathematica 81 (1949) 309.

[66] J.D. Lawson, J.L. Morris, The extrapolation of first order methods for parabolic partial differential equations. I, SIAM Journal on Numerical Analysis 15 (1978) 1212.

[67] J. Strutwolf, W.W. Schoeller, Digital simulation of potential step experiments using the extrapolation method, Electroanalysis 9 (1997) 1403.

[68] W.G. Bickley, Formulae for numerical differentiation, Mathematical Gazette 25 (1941) 19.

[69] C.F. Curtiss, J.O. Hirschfelder, Integration of stiff equations, Proceedings of the National Academy of Sciences of the United States of America 38 (1952) 235.

[70] J. Mocak, S.W. Feldberg, The Richtmyer modification of the fully implicit finite difference algorithm for simulations of electrochemical problems, Journal of Electroanalytical Chemistry 378 (1994) 31.

[71] D. Britz, Time shift artifacts and start-up protocols with the BDF method in electrochemical digital simulation, Computers and Chemistry 22 (1998) 237.

[72] J. Douglas Jr., H. Rachford Jr., On the numerical solution of heat conduction problems in two and three space dimensions, Transactions of the American Mathematical Society 82 (1956) 421.

[73] O. Østerby, Numerical Solution of Parabolic Equations, Tech. Rep. Daimi FN-65, Dept. of Computer Science, University of Aarhus, Denmark, 2008. Accessible at http://www.daimi.au.dk/oleby/notes/partial.pdf.

[74] http://www.mathworks.com/products/matlab/.

[75] A.R. Gourlay, J.L. Morris, The extrapolation of first order methods for parabolic partial differential equations. II, SIAM Journal on Numerical Analysis 17 (1980) 641.

[76] I.S. Duff, J.K. Reid, Some design features of a sparse matrix code, ACM Transactions on Mathematical Software 5 (1979) 18.

[77] http://hsl.rl.ac/archive/hslarchive.html.

[78] http://www.netlib.org/seispack/rgg.f.

[79] J. Crank, P. Nicolson, A practical method for numerical evaluation of solutions of partial differential equations of the heat-conduction type, Proceedings of the Cambridge Philological Society 43 (1947) 50, reprinted in Advances in Computational Mathematics 6 (1996) 207, with some slight changes to the list of references.

[80] D. Britz, O. Østerby, J. Strutwolf, Damping of Crank-Nicolson error oscillations, Computational Biology and Chemistry 27 (2003) 253.

[81] O. Østerby, Five ways of reducing the Crank-Nicolson oscillations, BIT Numerical Mathematics 43 (2003) 811. 


\section{Glossary}

ADI: Alternating direction implicit (method)

$B D F$ : backward differentiation formula (method)

AF: Amatore and Fosset (transformation)

BI: backward implicit (method)
$M F$ : Morse and Feshbach (transformation)

MWA: Michael, Wightman and Amatore (transformation)

$O A S$ : Oleinick, Amatore and Svir (transformation)

OC: orthogonal collocation (method)

pde: partial differential equation

$V B$ : Verbrugge and Baker (transformation) 